\title{
Use of nanotechnology in the diagnosis and treatment of coronavirus
}

\author{
Elham Maghareh Abed ${ }^{1}$, Seyedeh Mahsan Hoseini-Alfatemi², Hoda Sabati3, Mohammad Amin \\ Khajavi Gaskarei4, Kourosh Delpasand4, Marzie Ghasemi5,**
}

${ }^{1}$ Department of Microbiology, Faculty of Biological Sciences, Islamic Azad University, North Tehran Branch, Tehran, Iran ${ }_{2}^{2} P e d i a t r i c$ Infections Research Center, Research Institute for Children's Health, Shahid Beheshti University of Medical Sciences, Tehran, Iran

3Biotechnology and Biological Science Research Center, Shahid Chamran University of Ahvaz, Ahvaz, Iran 4Razi Clinical Research Development Unit, Guilan University of Medical Sciences, Rasht, Iran 5Department of Biochemistry, School of Medicine, Ahvaz Jundishapour University of Medical Sciences, Ahvaz, Iran

\begin{abstract}
Coronavirus is a beta virus that has caused a worldwide pandemic since December 2019. Many treatments such as antiviral drugs, immunosuppressive drugs, neutralizing antibodies, and monoclonal antibodies have been tested on coronavirus disease 2019 (COVID-19) that most of them were effective. Given that nanotechnology-based approaches have been successful in detection and treatment of viral systems such as human immunodeficiency virus (HIV), influenza A virus subtype H1N1 and Middle East respiratory syndrome coronavirus (MERS-CoV), they also seem to be effective in detecting and treating COVID-19. Nanotechnology is used in various methods for early and rapid diagnosis of the disease. Nanoparticles can be used in products for the diagnosis, treatment and prevention of COVID19. These substances are very effective in the controlled delivery of antiviral drugs and biomolecules and they are also used in the manufacture of personal safety equipment, widely, and the production of anti-virus coatings for surfaces, air filters and the production of vaccines. In general, nanomaterial can play an important role in controlling the disease, based on strategies to prevent the virus from entering the host cell, inhibiting virus replication, virus delivery systems, and nano-based vaccines. Nanotechnology is a multidisciplinary tool that can offer a variety of solutions based on disease prevention, diagnosis and treatment strategies.
\end{abstract}

Keywords: COVID-19, Nanotechnology, Coronavirus, Diagnosis, Treatment

\section{Introduction}

The corona pandemic that has occurred in the world since the last days of 2019 is related to a virus called coronavirus-2019 (COVID-19) and belongs to the beta virus family along with Severe acute respiratory syndrome coronavirus-2 (SARS-CoV-2) and Middle East respiratory syndrome coronavirus
(MERS-CoV) [1]. This virus has a single-stranded positive RNA genome [2]. The virus attaches to the human angiotensin converting enzyme-2 (ACE-2) and binds to the host cell. This enzyme is a receptor for the SARS-CoV-2 virus in the host cell [3]. They cause several diseases in humans and animals, including

\footnotetext{
${ }^{*}$ Corresponding author:

Marzie Ghasemi, MSc

Department of Biochemistry, School of Medicine,

Ahvaz Jundishapour University of Medical Sciences, Ahvaz, Iran

Tel/Fax: +989336357377

Email: marzieghasemi1985@gmail.com

https://orcid.org/oooo-0001-6753-8356
}

Received: February, 21, 2021

Accepted:, March, 13, 2021
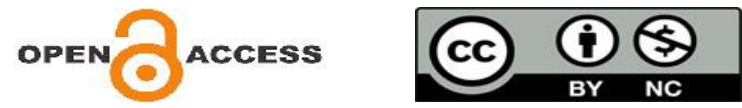
respiratory, intestinal, hepatic, and nervous systems [4].

The rate of human-to-human transmission of coronavirus is significantly high and causes a wide range of clinical manifestations in patients infected with the virus [5]. However, the reproductive number and transmission way are not yet completely understood, the routes of transmission with and without symptoms and the role of environmental factors should be better known. Widespread geographical epidemics and prevalence in large populations of the world, rapid transmission, recurrent infections and genomic alteration $[6,7]$, create difficult barriers to the development of ways to prevent, control, and treat this disease [8, 9].

A wide range of possible treatments is being tested. Based on the SARS-CoV-2 life cycle and its structure, the treatments proposed for Covid-19 have been antiviral drugs, immunosuppressive drugs, neutralizing and monoclonal antibodies [10], and vaccines.

Antiviral drugs effect by inhibiting ribonucleic acid (RNA) polymerase, viral protease, membrane occlusion, and widespread of antiviral effects [10]. Currently, oxygen therapy and the use of the respiratory tract play a major role in the treatment of COVID-19 [11].

Recent clinical results for antiviral drugs have not been very satisfactory [12]. Some drugs have also been suggested to reduce the damage caused by SARS-CoV2 to thelungs [8]. In some cases, plasma therapy is also suggested [13]. This method of treatment is used in patients who are not able to produce enough antibodies and is a safe and effective method and seems to reduce mortality [14].

Scientists believe nanotechnology-based approaches could help against covid-19. Based on previous studies, nanotechnology can be effective in identifying viral systems and overcome the limitations of conventional methods of viral disease strategies $[15$, 16].

In this study, in addition to reviewing the effects of nanotechnology in the fight against other viral diseases, the proposed nanotechnology-based solutions in the diagnosis, treatment, and prevention of environmental pollution caused by covid-19 are examined.
2. Application of nanotechnology in the detection and treatment of viral infections

According to previous research, nanotechnology has been considered in the process of diagnosis and treatment of many viral diseases. The use of luminescence electrochemical biosensors for the selective detection of avian influenza virus (H9N2AIV) [17] and the use of magnetic nanoparticles (NPs) for the detection of influenza A virus subtype H1N1 virus [18] are examples of nanoparticles applications in virus detection. The use of poly vinyl pyrrolidone (PVP) coated AgNPs (30-50 nm) on HIV strains has also been shown to have antiviral activity [19].

Curcumin nanoparticles also have high antiviral properties against the respiratory syncytial virus (RSV) [20]. Dendrimers have also been extensively studied for human immunodeficiency virus (HIV) disinfection applications. These nanoparticles suppress virus replication [21]. Lipid-based nanoparticles have previously been tested for the treatment of HIV, herpes, hepatitis B (HBV), and hepatitis $\mathrm{C}(\mathrm{HCV})$ viruses. These nanoparticles can encapsulate different classes of antiviral drugs and are a mediator of drug delivery to the target tissue or cell, as well as modulating specific biological responses [22].

Aluminum nanoparticles are another class of metal structures that can be used in vaccines for respiratory viruses such as MERS-CoV and SARSCoV-2. These compounds elicit cellular and humoral immunity responses [23]. Unsaturated liposomes also have an inhibitory effect against HBV, HCV, and HIV [22].

\section{SARS-CoV-2 and nanotechnology insights}

Nanotechnology-based treatment strategies are a promising way to overcome the current limitations in the prevention, diagnosis, and treatment of Covid-19 [24]. Nanomaterials can be used for disease diagnosis to develop simpler, cheaper, and faster methods. In therapy, nanosystems can be used in the controlled delivery of antiviral drugs and biomolecules to the lungs and target tissues that are used to prevent virus replication or inactivation of viral particles [25]. There are successful prospects in building safe personal protective equipment [25] and producing vaccines or immune modulators using nanotechnology [16]. 


\section{Detection of SARS-CoV-2 with nanotechnology}

Currently, the only way to diagnose Covid-19 infection is to test for nucleic acid by reverse transcription polymerase chain reaction (RT-PCR). But this method also has limitations; it is not able to diagnose asymptomatic patients, not all health care centers, especially in non-urban environments, are equipped with sufficient PCR infrastructure. While accurate and early diagnosis of the disease is a vital requirement for essential care. Nanotechnology can meet these demands through nucleic acid testing methods, point-of-care testing (POCT), electrochemical sensors, and biosensors [15].

In the nucleic acid test method, the nucleic acid is amplified in nucleic acid isothermal conditions. This method is very sensitive, rapid, and specific [26]. Nanomaterials exhibit electrical and optical properties that have been extensively applied in the development of diagnostic methods such as point-of-care testing (POCT) biosensors.

These substances can improve the sensitivity of diagnosis, rapid isolation of patients, and facilitate the treatment process [24]. Different types of nanomaterials include; nanoparticles, nanoclusters, quantum dots, carbon nanotubes, nanocomposites, etc that have been used to increase the performance of biosensors. Recently, deoxyribonucleic acid (DNA) sensor nanomaterials have been developed for simple, rapid, selective, low-cost, and sensitive detection of infectious diseases [27]. Biosensors are color-coded test points that can detect contaminated specimens through simple color changes that are visible to the naked eye [15].

A sample of these POCTs has been generated using gold nanoparticles to detect the MERS-CoV virus [28]. In another method, reverse transcription Loop-Mediated Isothermal Amplification (RT-LAMP) with nano-based biosensors has been used to detect COVID-19 [29].

Electrochemical sensors are effective in detecting viruses due to their high sensitivity and the possibility of shrinkage. Gold nanoparticles (AuNPs) are one of the most attractive nanomaterials because they have electronic optical properties that can be detected by a variety of biosensors, especially those based on colorimetric, electrochemical [30]. The use of AuNPs in these sensors is much more effective in detecting viruses by immobilizing biomolecules while maintaining their function [31].

\section{Treatment of SARS-CoV-2 with nanotechnology}

The specific antiviral drug for SARS-CoV-2 is not currently available to the public [32]. Production of these drugs is a time-consuming process that must be monitored and tested for safety before use [33]. On the other hand, increasing virus resistance is another limitation of the production and use of antiviral drugs [15]. In some studies, nanotechnology has been widely applied in the treatment of this disease. Nanotechnology through methods such as; preventing viruses from entering host cells, inhibiting virus replication, virus transmission systems, and nanobased vaccines is probably successful in treating the disease [15].

Also, many of the proposed treatments for covid19 infection that are spreading, use nano-based methods such as; SARS-CoV-2 virus-like particles by using iBio's Fast Pharming technology[15].

Nanoparticles (NP) (organic and inorganic) have been considered for some of their properties. Inorganic NPs (INPs) have properties such as luminescence, adjustable size, shape, composition, high surface-to-volume ratio, and the ability to expose multiple reciprocal locations on the surface. The most common types of INPs are mesoporous silica NPs, iron oxide NPs and metal NPs (gold, silver). Organic NPs include; Carbon nanotubes and graphene nanoparticles are polymeric and lipid NPs, dendrimers, extracellular vesicles (or exosomes), liposomes, and nanomolecules. Organic nanoparticles are being noticed because of their potential sitespecific targeting, drug release control, biodegradation, biocompatibility, and non-toxicity [34].

\section{Pharmacological treatment}

The first step in starting a viral infection is the virus's attachment to the host. Nanoparticles have the ability to prevent the virus from adherent to the host cell and entering the cell [35]. One way to use nanoparticles to prevent the virus from entering the host cell is to use carbon quantum dots that if combined with boronic acid show stronger antiviral activity [36]. The use of gold nano rods in antiviral therapy for MERS has also been successful [37]. 
Second, if the virus enters the host cell, the spread of infection can be prevented with nanoparticles, mainly as carriers for the delivery of antiviral molecules.

Higher specificity, improvement of drug solubility, the combination of different drug molecules in one particle, and reduction of toxicity for the host are the advantages of nanoparticles in this field [15]. Some nanoparticles, such as $\mathrm{Ag}_{2} \mathrm{~S}$ nanoclusters, can prevent the virus from replicating in the host body. These nanoparticles also play a role in enhancing the expression of pro-inflammatory cytokines, which are effective against viral infections [38]. Zinc oxide ( $\mathrm{ZnO})$ nanoparticles have similar activity and effective against $\mathrm{H} 1 \mathrm{~N} 1$ influenza virus infection [39] and it also seems to be involved in inhibiting the proliferation of SARS-CoV-2 [40].

Traditional methods of prescribing antiviral drugs have limitations such as poor bioavailability, systemic toxicity, susceptibility to in vivo destruction, and short half-life.

In contrast, nanotechnology drugs have advantages such as higher bioavailability, lower toxicity, protection against degradation, improved circulating half-life, reduced effective dose of the drug, the ability to cross biological barriers [41], and tissue or cellular targeting [42].

Also, nanocarriers provide the ability to control drug release in the target tissue, improve biocompatibility and reduce drug toxicity in healthy tissues by confining drugs [24]. Nano compounds such as micelles, microspheres, liposomes, and dendrimers have been used for better and targeted delivery of antiviral drugs such as efavirenz, zidovudine, and acyclovir [42].

\section{Prevention of SARS-CoV-2 with nanotechnology}

7.1 Improving personal protective equipment (PPE) using nanotechnology

In cases where a definitive cure for COVID-19 has not yet been discovered, prevention is especially important in vulnerable people such as the elderly, those with immunodeficiency, and at-risk individuals include healthcare professionals [43]. Although observing physical distance is known as the first step but, it is difficult to implement in the healthcare staff. Therefore, equipping these people with highly efficient safety and PPE is a very necessary step [15].
7.2 Environmental, surface disinfectants, and healthcare

Studies have shown that the SARS-CoV-2 virus remains on the surface for a long time [44, 45], so even the PPE kits used may be infected with the virus [46]. Therefore, the production of anti-virus coatings on surfaces and air filtration devices can help reduce infection.

Nanomaterials have the ability to prevent viral contamination by air and contact with contaminated surfaces and have the ability to sterilize protective equipment, especially in hospital settings [24]. Creating self-disinfecting surfaces is another strategy to prevent the spread of COVID-19.

Coating surfaces using nanomaterials such as silver, zinc oxide, and copper NPs is one of these solutions. These compounds oxidize and release ions with antimicrobial properties [25, 47]. According to studies, controlled and long-term diffusion of ions in some metals, such as copper, modulates the antiviral properties of surfaces [48].

These nanoparticles are loaded into a polymer matrix and provide an effective antiviral coating on surfaces [15]. This method can also be applied to polypropylene masks. This method has already been used in the preparation of protective masks against influenza viruses. This was done by depositing a coating of silver or silica nanocoating composite on face masks [49]. Graphene in face masks can also sterilize these and allow them to be reused [50]. This coating is also suitable for use on surfaces in public places [51].

There are several products made of nanocomposites that have antimicrobial activity and are used in the disinfection of surfaces. CAC-717 is a new disinfectant consisting of calcium hydrogen carbonate mesoscopic crystals that are a compound containing mesostructured NPs and are involved in inactivating enveloping [52] and non-enveloping [53] viruses.

Tungsten carbide nanoparticles also have extensive antiviral activity [54]. AgNPs also have various medical, environmental, and industrial applications as disinfectants [55]. These compounds have antiviral activity against several types of viruses, including RSV, HBV, and HIV [56]. These nanoparticles can also be used in air filters [55] and clothing fabrics [57]. 


\section{Nano-based vaccines}

Many efforts are currently underway to develop the COVID-19 vaccine, including clinical trials. Nanotechnology approaches can help develop nanobased vaccines. The apparent similarity of SARS-CoV2 with other viruses (mainly SARS-CoV-2 and MERS$\mathrm{CoV}$ ) and the therapeutic progress and challenges of SARS-CoV-2 infection with other infectious diseases as well as oncological research are not significantly different [58], and it can help a lot in making a successful COVID-19 vaccine [59].

Vaccine production has significant similarities in the fight against SARS, MERS coronaviruses [16]. On the other hand, Conventional vaccines have problems such as the risk of reversal of viral virulence (live attenuated virus vaccines), weak immune response (inactivated viruses), and limited immunogenicity (subunit vaccines) [15].

Another advantage of vaccine nanocarriers is their size. Many biological systems, such as viruses, including the SARS-CoV-2 virus and some proteins, are nano-scale [16]. In this way, nanoparticles can be loaded with a wide range of antigenic components which is very important in the production of vaccines [6o]. Nanoparticles can also target both humoral immune systems and cell-mediated immunity [61]. Modulation of antigen-presenting cells (APCs) using nanoparticles is of particular importance in vaccine fabrication [62]. Organic nanoparticles such as liposomes, lipid-based NPs, and polymeric NPs are significant in vaccine formulations [24]. Also, biopolymer-based NPs, like protein-based NPs, have low toxicity and biodegradability [63]. AuNPs, as another nanosystem for vaccine production, have been studied on more than 45 pathogens in various sizes, shapes, and functions [64].

\section{Conclusion}

The outbreak of the COVID-19 virus over the past year has led to the infection of a large population of the world and high mortality. As a result, rapid progress in discovering ways to diagnose, treat, and control the disease is a necessity. Early and rapid diagnosis of the disease, rapid and effective treatment measures, the use of appropriate and safe equipment are the most important strategies to control the disease. Currently, the diagnosing method of this disease is timeconsuming and it is not applicable in public populations and far from urban health centers. On the other hand, many therapeutic approaches to this disease are based on symptomatic treatment and are performed using antiviral drugs previously used in other viral diseases. These drugs prevent the virus from being absorbed into the tissue and block the activity of proteases in infected cells. However, this strategy only reduces the number of viruses and the symptoms. According to reports, the most important barrier to the use of these drugs is their delivery to target cells and tissues in the respiratory tract, which reduces their effectiveness.

Use methods based on nanotechnology is one of the best ways to overcome these barriers. Due to the aerodynamic size of the nanoparticles, they can be effective in predicting the treatment process. Also, the lack of an approved vaccine with high efficacy and low side effects is another problem and obstacle in controlling the disease. The unpredictable and unknown nature of the disease and the similarity of the specific properties of COVID-19 and the physicochemical properties of nanosystems have led to the discussion of solutions based on new technologies.

This study attempts to review possible strategies for the prevention, diagnosis, and treatment of COVID-19 by focusing on current experiences and knowledge about nanotechnology and its successful measures in curing other viral diseases. The use of proprietary antiviral drugs, animal models, in vitro and in vivo studies to help screen for antiviral drugs, prevent disease transmission, and produce vaccines with minimal side effects and high efficacy as soon as possible will determine the prospects of disease control. In summary, nanotechnology is a powerful multidisciplinary tool that has a variety of approaches and strategies to improve the development of coronavirus control and treatment.

\section{Author Contributions}

EM, MH, MG conceptualized and designed the study. EM, MH, MK, KD data collection. EM, MH, HS, MK, KD, MG drafted the manuscript, and revised the final version of manuscript.

\section{Conflict of Interests}

The authors declare that they have no conflicts of interest.

\section{Ethical declarations}


Not applicable.

\section{Financial Support}

Self-funding.

\section{References}

1. Rehman SU, Shafique L, Ihsan A, Liu Q. Evolutionary Trajectory for the Emergence of Novel Coronavirus SARS-CoV-2. Pathogens. 2020; 9(3):240.

2. Ruiz-Hitzky E, Darder M, Wicklein B, Ruiz-Garcia C, MartínSampedro R, Del Real G, et al. Nanotechnology Responses to COVID-19. Adv Healthc Mater. 2020; 9(19):e2000979.

3. Ou X, Liu Y, Lei X, Li P, Mi D, Ren L, et al. Characterization of spike glycoprotein of SARS-CoV-2 on virus entry and its immune cross-reactivity with SARS-CoV. Nat Commun. 2020; 11(1):1620.

4. Zumla A, Chan JF, Azhar EI, Hui DS, Yuen KY. Coronaviruses drug discovery and therapeutic options. Nat Rev Drug Discov. 2016; 15(5):327-47.

5. Khan M, Adil SF, Alkhathlan HZ, Tahir MN, Saif S, Khan M, et al. COVID-19: A Global Challenge with Old History, Epidemiology and Progress So Far. Molecules. 2020; 26(1):39.

6. Forster P, Forster L, Renfrew C, Forster M. Phylogenetic network analysis of SARS-CoV-2 genomes. Proc Natl Acad Sci USA. 2020; 117(17):9241-3.

7. Guan WJ, Ni ZY, Hu Y, Liang WH, Ou CQ, He JX, et al. Clinical Characteristics of Coronavirus Disease 2019 in China. N Engl J Med. 2020; 382(18):1708-20.

8. Tang Z, Zhang X, Shu Y, Guo M, Zhang H, Tao W. Insights from nanotechnology in COVID-19 treatment. Nano Today. 2021; 36:101019.

9. Walls AC, Park YJ, Tortorici MA, Wall A, McGuire AT, Veesler D. Structure, Function, and Antigenicity of the SARS-CoV-2 Spike Glycoprotein. Cell. 2020; 181(2):281-92.e6.

10. Tian X, Li C, Huang A, Xia S, Lu S, Shi Z, et al. Potent binding of 2019 novel coronavirus spike protein by a SARS coronavirusspecific human monoclonal antibody. Emerg Microbes Infect. 2020; 9(1):382-5.

11. Mizumoto K, Kagaya K, Zarebski A, Chowell G. Estimating the asymptomatic proportion of coronavirus disease 2019 (COVID-19) cases on board the Diamond Princess cruise ship, Yokohama, Japan, 2020. Euro Surveill. 2020; 25(10):2000180.

12. Grein J, Ohmagari N, Shin D, Diaz G, Asperges E, Castagna A, et al. Compassionate Use of Remdesivir for Patients with Severe Covid-19. N Engl J Med. 2020; 382(24):2327-36.

13. Bhavana V, Thakor P, Singh SB, Mehra NK. COVID-19: Pathophysiology, treatment options, nanotechnology approaches, and research agenda to combating the SARS-CoV2 pandemic. Life Sci. 2020; 261:118336.

14. UK researchers launch trial of blood plasma therapy for Covid19. UK researchers launch trial of blood plasma therapy for Covid192020 [updated May 4th, 2020]. Available at: https://www.clinicaltrialsarena.com/news/uk-blood-plasmacovid-19-trial/.

15. Jindal S, Gopinath P. Nanotechnology based approaches for combatting COVID-19 viral infection. Nano Express. 2020 1(2).

16. Chauhan G, Madou MJ, Kalra S, Chopra V, Ghosh D, MartinezChapa SO. Nanotechnology for COVID-19: Therapeutics and Vaccine Research. ACS Nano. 2020; 14(7):7760-82.
17. Luo F, Long $\mathrm{C}$, Wu Z, Xiong $\mathrm{H}$, Chen $\mathrm{M}$, Zhang $\mathrm{X}$, et al. Functional silica nanospheres for sensitive detection of H9N2 avian influenza virus based on immunomagnetic separation. Sens Actuators B Chem. 2020; 310:127831.

18. Singh R, Hong S, Jang J. Label-free detection of influenza viruses using a reduced graphene oxide-based electrochemical immunosensor integrated with a microfluidic platform. Sci Rep. 2017; 7:42771.

19. Lara HH, Ayala-Nuñez NV, Ixtepan-Turrent L, RodriguezPadilla C. Mode of antiviral action of silver nanoparticles against HIV-1. J Nanobiotechnology. 2010; 8:1.

20. Yang X, Li C, Huang C. Curcumin modified silver nanoparticles for highly efficient inhibition of respiratory syncytial virus infection. Nanoscale. 2016; 8(5):3040-8.

21. Mhlwatika Z, Aderibigbe B. Application of dendrimers for the treatment of infectious diseases. Molecules. 2018; 23(9):2205.

22. Pollock S, Nichita N, Böhmer A, Radulescu C, Dwek R, Zitzmann N. Polyunsaturated liposomes are antiviral against hepatitis $\mathrm{B}$ and $\mathrm{C}$ viruses and HIV by decreasing cholesterol levels in infected cells. Proc Natl Acad Sci U S A. 2010; 107(40):17176-81. 23. Wang N, Wei C, Zhang Z, Liu T, Wang T. Aluminum Nanoparticles Acting as a Pulmonary Vaccine Adjuvant-Delivery System (VADS) Able to Safely Elicit Robust Systemic and Mucosal Immunity. J Inorg Organomet Polym Mater. 2020; 30:4203-17. 24. Cardoso VMdO, Moreira BJ, Comparetti EJ, Sampaio I, Ferreira LMB, Lins PMP, et al. Is Nanotechnology Helping in the Fight Against COVID-19? Frontiers in Nanotechnology. 2020; 2(4):588915.

25. Weiss C, Carriere M, Fusco L, Capua I, Regla-Nava JA, Pasquali $\mathrm{M}$, et al. Toward Nanotechnology-Enabled Approaches against the COVID-19 Pandemic. ACS Nano. 2020; 14(6):6383-406.

26. Nurul Najian AB, Engku Nur Syafirah EA, Ismail N, Mohamed $\mathrm{M}$, Yean CY. Development of multiplex loop mediated isothermal amplification (m-LAMP) label-based gold nanoparticles lateral flow dipstick biosensor for detection of pathogenic Leptospira. Anal Chim Acta. 2016; 903:142-8.

27. Mozhgani SH, Kermani HA, Norouzi M, Arabi M, Soltani S. Nanotechnology based strategies for HIV-1 and HTLV-1 retroviruses gene detection. Heliyon. 2020; 6(5):e04048.

28. Kim H, Park M, Hwang J, Kim JH, Chung DR, Lee KS, et al. Development of Label-Free Colorimetric Assay for MERS-CoV Using Gold Nanoparticles. ACS Sens. 2019; 4(5):1306-12.

29. Zhu X, Wang X, Han L, Chen T, Wang L, Li H, et al. Multiplex reverse transcription loop-mediated isothermal amplification combined with nanoparticle-based lateral flow biosensor for the diagnosis of COVID-19. Biosens Bioelectron. 2020; 166:112437.

30. Huang Y, Xu T, Wang W, Wen Y, Li K, Qian L, et al. Lateral flow biosensors based on the use of micro- and nanomaterials: a review on recent developments. Mikrochim Acta. 2019; 187(1):70.

31. Layqah LA, Eissa S. An electrochemical immunosensor for the corona virus associated with the Middle East respiratory syndrome using an array of gold nanoparticle-modified carbon electrodes. Mikrochim Acta. 2019; 186(4):224.

32. Amanat F, Krammer F. SARS-CoV-2 Vaccines: Status Report. Immunity. 2020; 52(4):583-9.

33. Chen WH, Strych U, Hotez PJ, Bottazzi ME. The SARS-CoV-2 Vaccine Pipeline: an Overview. Curr Trop Med Rep. 2020:1-4. 
34. Kerry RG, Malik S, Redda YT, Sahoo S, Patra JK, Majhi S. Nano-based approach to combat emerging viral (NIPAH virus) infection. Nanomedicine. 2019; 18:196-220.

35. Khanal M, Vausselin T, Barras A, Bande O, Turcheniuk K, Benazza M, et al. Phenylboronic-acid-modified nanoparticles: potential antiviral therapeutics. ACS Appl Mater Interfaces. 2013; 5(23):12488-98.

36. Łoczechin A, Séron K, Barras A, Giovanelli E, Belouzard S, Chen YT, et al. Functional Carbon Quantum Dots as Medical Countermeasures to Human Coronavirus. ACS Appl Mater Interfaces. 2019; 11(46):42964-74.

37. Huang X, Li M, Xu Y, Zhang J, Meng X, An X, et al. Novel Gold Nanorod-Based HR1 Peptide Inhibitor for Middle East Respiratory Syndrome Coronavirus. ACS Appl Mater Interfaces. 2019; 11(22):19799-807.

38. Du T, Liang J, Dong N, Lu J, Fu Y, Fang L, et al. GlutathioneCapped $\mathrm{Ag}(2) \mathrm{S}$ Nanoclusters Inhibit Coronavirus Proliferation through Blockage of Viral RNA Synthesis and Budding. ACS Appl Mater Interfaces. 2018; 10(5):4369-78.

39. Ghaffari H, Tavakoli A, Moradi A, Tabarraei A, BokharaeiSalim F, Zahmatkeshan M, et al. Inhibition of H1N1 influenza virus infection by zinc oxide nanoparticles: another emerging application of nanomedicine. J Biomed Sci. 2019; 26(1):70.

40. Razzaque MS. COVID-19 Pandemic: Can Maintaining Optimal Zinc Balance Enhance Host Resistance? Tohoku J Exp Med. 2020; 251(3):175-81.

41. Kobayashi K, Wei J, Iida R, Ijiro K, Niikura K. Surface engineering of nanoparticles for therapeutic applications. Polymer J. 2014; 46(8):460-8.

42. Milovanovic M, Arsenijevic A, Milovanovic J, Kanjevac T, Arsenijevic N. Chapter 14 - Nanoparticles in Antiviral Therapy. In: Grumezescu AM, editor. Antimicrobial Nanoarchitectonics: Elsevier; 2017. p. 383-410.

43. Chang D, Xu H, Rebaza A, Sharma L, Dela Cruz CS. Protecting health-care workers from subclinical coronavirus infection. Lancet Respir Med. 2020; 8(3):e13.

44. Warnes SL, Little ZR, Keevil CW. Human Coronavirus 229E Remains Infectious on Common Touch Surface Materials. mBio. 2015; 6(6):e01697-15.

45. Kampf G, Todt D, Pfaender S, Steinmann E. Persistence of coronaviruses on inanimate surfaces and their inactivation with biocidal agents. J Hosp Infect. 2020; 104(3):246-51.

46. Ong S, Tan Y, Chia P, Lee T, Ng O, Wong M, et al. Air, surface environmental, and personal protective equipment contamination by severe acute respiratory syndrome coronavirus 2 (SARS-CoV2) from a symptomatic patient. JAMA. 2020; 323(16):1610-2.

47. Murphy F, Tchetchik A, Furxhi I. Reduction of Health CareAssociated Infections (HAIs) with Antimicrobial Inorganic Nanoparticles Incorporated in Medical Textiles: An Economic Assessment. Nanomaterials (Basel). 2020; 10(5):999.

48. Palza H, Nuñez M, Bastías R, Delgado K. In situ antimicrobial behavior of materials with copper-based additives in a hospital environment. Int J Antimicrob Agents. 2018; 51(6):912-7.
49. Iyigundogdu ZU, Demir O, Asutay AB, Sahin F. Developing Novel Antimicrobial and Antiviral Textile Products. Appl Biochem Biotechnol. 2017; 181(3):1155-66.

50. Akhavan O, Choobtashani M, Ghaderi E. Protein degradation and RNA efflux of viruses photocatalyzed by graphene-tungsten oxide composite under visible light irradiation. J Phys Chem C. 2012; 116(17):9653-9.

51. Balagna C, Perero S, Percivalle E, Nepita EV, Ferraris M. Virucidal effect against coronavirus SARS-CoV-2 of a silver nanocluster/silica composite sputtered coating. Open Ceram. 2020; 1:100006.

52. Nakashima R, Kawamoto M, Miyazaki S, Onishi R, Furusaki K, Osaki M, et al. Evaluation of calcium hydrogen carbonate mesoscopic crystals as a disinfectant for influenza A viruses. J Vet Med Sci. 2017; 79(5):939-42.

53. Sakudo A, Yamashiro R, Haritani M, Furusaki K, Onishi R, Onodera T. Inactivation of Non-Enveloped Viruses and Bacteria by an Electrically Charged Disinfectant Containing Meso-Structure Nanoparticles via Modification of the Genome. Int $\mathrm{J}$ Nanomedicine. 2020; 15:1387-95.

54. Pfaff F, Glück B, Hoyer T, Rohländer D, Sauerbrei A, Zell R. Tungsten carbide nanoparticles show a broad spectrum virucidal activity against enveloped and nonenveloped model viruses using a guideline-standardized in vitro test. Lett Appl Microbiol. 2019; 69:302-9.

55. Deshmukh SP, Patil SM, Mullani SB, Delekar SD. Silver nanoparticles as an effective disinfectant: A review. Mater Sci Eng C Mater Biol Appl. 2019; 97:954-65.

56. Galdiero S, Falanga A, Vitiello M, Cantisani M, Marra V, Galdiero M. Silver nanoparticles as potential antiviral agents. Molecules. 2011; 16(10):8894-918.

57. Noor N, Mutalik S, Younas MW, Chan CY, Thakur S, Wang F, et al. Durable Antimicrobial Behaviour from Silver-Graphene Coated Medical Textile Composites. Polymers (Basel). 2019; 11(12):2000.

58. Kostarelos K. Nanoscale nights of COVID-19. Nat Nanotechnol. 2020; 15(5):343-4.

59. Lurie N, Saville M, Hatchett R, Halton J. Developing Covid-19 Vaccines at Pandemic Speed. N Engl J Med. 2020; 382(21):196973.

60. Park YM, Lee SJ, Kim YS, Lee MH, Cha GS, Jung ID, et al. Nanoparticle-based vaccine delivery for cancer immunotherapy. Immune Netw. 2013; 13(5):177-83.

61. Graham RL, Becker MM, Eckerle LD, Bolles M, Denison MR, Baric RS. A live, impaired-fidelity coronavirus vaccine protects in an aged, immunocompromised mouse model of lethal disease. Nat Med. 2012; 18(12):1820-6.

62. Steinman RM, Banchereau J. Taking dendritic cells into medicine. Nature. 2007; 449(7161):419-26.

63. Lohcharoenkal W, Wang L, Chen YC, Rojanasakul Y. Protein nanoparticles as drug delivery carriers for cancer therapy. Biomed Res Int. 2014; 2014:180549.

64. Dykman LA. Gold nanoparticles for preparation of antibodies and vaccines against infectious diseases. Expert Rev Vaccines. 2020; 19(5):465-77. 